\title{
ASSUMED PDF MODELING IN ROCKET COMBUSTOR SIMULATIONS
}

\author{
M. Lempke, P. Gerlinger, and M. Aigner \\ University of Stuttgart, Institute of Combustion Technology \\ 38-40 Pfaffenwaldring, Stuttgart 70569, Germany
}

\begin{abstract}
In order to account for the interaction between turbulence and chemistry, a multivariate assumed PDF (Probability Density Function) approach is used to simulate a model rocket combustor with finite-rate chemistry. The reported test case is the PennState preburner combustor with a single shear coaxial injector. Experimental data for the wall heat flux is available for this configuration. Unsteady RANS (Reynolds-averaged Navier-Stokes) simulation results with and without the assumed PDF approach are analyzed and compared with the experimental data. Both calculations show a good agreement with the experimental wall heat flux data. Significant changes due to the utilization of the assumed PDF approach can be observed in the radicals, e.g., the $\mathrm{OH}$ mass fraction distribution, while the effect on the wall heat flux is insignificant.
\end{abstract}

\section{INTRODUCTION}

Due to the high costs for ground testing, it is inevitable to rely as much as possible on CFD (Computational Fluid Dynamics) simulations in the design process of rocket injectors and combustors. However, even the capabilities of high-fidelity approaches like LES (Large Eddy Simulation) have to be improved in order to be accurate enough, to have a quantitative influence on the injector design process [1].

Direct Numerical Simulation (DNS) approaches would yield much better results, but are computationally still more expensive and, therefore, restricted to the simulation of single injector near-field phenomena assuming nonreacting flows or using simple combustion models. Ideally, however, a CFD tool should be able to simulate complete combustor geometries with up to several hundred injector heads including the nozzle in an acceptable timespan and at acceptable cost. Accordingly, RANS methods are still the design tools of choice in industrial applications where time-averaged quantities often are sufficient.

This is an Open Access article distributed under the terms of the Creative Commons Attribution License 2.0, which permits unrestricted use, distribution, and reproduction in any medium, provided the original work is properly cited. 
However, recent work has shown the limitations of RANS methods when it comes to predicting the wall heat flux in a model rocket combustor with shear coaxial injectors $[1-3]$.

Unsteady RANS simulations, on the other hand, have demonstrated the capability to yield quite useful results for this kind of combustor [2, 3]. However, they are per se not able to reproduce the strong nonlinearity of the chemical source terms arising from turbulence-chemistry interaction. Instead, chemistry is usual treated in a "laminar" way. The use of PDF methods is a promising approach to tackle this problem. It appears to be the natural choice to treat statistical fluctuations in the flowfield with probabilistic methods. The two major groups of PDF methods are the assumed PDF approaches and the PDF transport equation methods.

The more sophisticated approach that does not predefine the shape of the $\mathrm{PDF}$ is the solution of a PDF transport equation. It has the advantage of treating convection and finite-rate nonlinear chemistry without modeling, if the PDF comprises all relevant variables. Those variables are the velocity vector for the convection and the thermochemical variables $\left(h\right.$ and $\left.Y_{i}\right)$ for the source term due to chemical reactions. This approach is called joint velocity-scalar PDF. However, there remain closure problems due to the fluctuating pressure gradient and due to molecular transport [4] which have to be modeled. Furthermore, the transported PDF approaches require extensive computational resources.

In assumed PDF methods, the shape of the PDF is mathematically predefined. Thus, the main focus in these methods is to find the functions that are as simple as possible from a mathematical point of view but flexible enough to be able to approximate realistic PDF shapes observed in experiments. Due to the comparatively low computational costs, assumed PDF methods are widely used and have proven the capability to produce useful results over a wide variety of combustion conditions $[5,6]$.

This paper aims to investigate the feasibility of the assumed PDF approach under rocket combustion conditions while only moderately increasing the computational cost.

\section{NUMERICAL CODE}

For all simulations in the present paper, the scientific TASCOM3D (Turbulent All Speed Combustion Multigrid three-dimensional) code is used. However, it has to be noted that the presented simulations were performed in a two-dimensional (2D) axisymmetric manner to reduce computational time. The code solves the conservation equations for mass, momentum, energy, and species as well as equations for turbulence closure. Several two-equation turbulence models are imple- 
mented in the code. However, the presented results are obtained with the $k-\omega$ model proposed by Wilcox [7]. When the assumed PDF module is activated, two additional equations for the temperature variance and the sum of the species mass fraction variances have to be solved.

The complete set of equations in three-dimensional (3D) conservative form can be written as

$$
\frac{\partial \mathbf{Q}}{\partial t}+\frac{\partial\left(\mathbf{F}-\mathbf{F}_{\nu}\right)}{\partial x}+\frac{\partial\left(\mathbf{G}-\mathbf{G}_{\nu}\right)}{\partial y}+\frac{\partial\left(\mathbf{H}-\mathbf{H}_{\nu}\right)}{\partial z}=\mathbf{S}
$$

with the vector of conservative variables

$$
\mathbf{Q}=\left[\bar{\rho}, \bar{\rho} \tilde{u}, \bar{\rho} \tilde{v}, \bar{\rho} \tilde{w}, \bar{\rho} \tilde{E}, \bar{\rho} k, \bar{\rho} \omega, \bar{\rho} \sigma_{T}, \bar{\rho} \sigma_{Y}, \bar{\rho} \mathbf{Y}\right]^{\mathrm{T}} .
$$

The variables in the variable vector $\mathbf{Q}$ are the density $\bar{\rho}$ (averaged); the velocity components (Favre averaged) $\tilde{u}, \tilde{v}$, and $\tilde{w}$; the total specific energy $\tilde{E}$; the turbulence variables $k$ and $\omega=\epsilon / k$ (where $k$ is the kinetic energy and $\epsilon$ is the dissipation rate of $k$ ); the variance of the temperature $\sigma_{T}$; and the sum of the species mass fraction variances $\sigma_{Y}$. The vector $\mathbf{Y}$ contains the species mass fractions $\tilde{Y}_{i}$ for $i=1,2, \ldots, N_{k}-1$ and $N_{k}$ is the total number of gaseous species which are assumed to be ideal gases. Since the set of equations includes the conservation of mass, $N_{k}-1$ species transport equations have to be solved, only. In Eq. (1), the vectors $\mathbf{F}, \mathbf{G}$, and $\mathbf{H}$ specify the inviscid fluxes and $\mathbf{F}_{\nu}, \mathbf{G}_{\nu}$ and $\mathbf{H}_{\nu}$ the viscous fluxes in $x$-, $y$-, and $z$-directions, respectively.

The momentary chemical production rate of species $i$ is defined by

$$
S_{i}=M_{i} \sum_{r=1}^{N_{r}}\left[\left(\nu_{i, r}^{\prime \prime}-\nu_{i, r}^{\prime}\right)\left(k_{f r} \prod_{l=1}^{N_{k}} c_{l}^{\nu_{l, r}^{\prime}}-k_{b r} \prod_{l=1}^{N_{k}} c_{l}^{\nu_{l, r}^{\prime \prime}}\right)\right]
$$

where $k_{f_{r}}$ and $k_{b_{r}}$ are the forward and backward rate constants of reaction $r$; $M_{i}$ is the molecular weight; and $c_{i}$ is the concentration of species $i$. Finally, $\nu_{i, r}^{\prime}$ and $\nu_{i, r}^{\prime \prime}$ denote the stoichiometric coefficients of species $i$ in reaction $r$. When chemistry is treated in a laminar way, mean values of the species concentration and temperature are used to calculate the reaction rates in Eq. (2). The PDF approaches, on the other hand, incorporate the turbulent fluctuations of these quantities.

Thus, the averaged chemical source term can be obtained by integrating the instantaneous source terms using a PDF according to

$$
\bar{S}_{i}=\left\langle S_{i}\right\rangle=\int_{\hat{T}} \int_{\hat{c}_{1}} \ldots \int_{\hat{c}_{N_{k}}} S_{i}\left(\hat{T}, \hat{c}_{1}, \ldots, \hat{c}_{N_{k}}\right) P\left(\hat{T}, \hat{c}_{1}, \ldots, \hat{c}_{N_{k}}\right) d \hat{T} d \hat{c}_{1} \cdots d \hat{c}_{N_{k}} .
$$

Presuming statistical independence between species and temperature fluctuations and keeping the density constant at its averaged value, the PDF can be 
replaced by the product of a temperature and a species PDF, $P_{T}$ and $P_{Y}$, respectively. In the present case, the assumed PDF approach uses mathematically predefined PDFs for the temperature and the species mass fraction. A widely used function for the temperature PDF $P_{T}$ is the clipped Gaussian distribution:

$$
\begin{array}{r}
P_{T}(\hat{T})=\frac{1}{\sqrt{2 \pi \sigma_{T}}} \exp \left(-\frac{(\hat{T}-\tilde{T})^{2}}{2 \sigma_{T}}\right) \\
\left(\theta\left(\hat{T}-T_{\min }\right)-\theta\left(\hat{T}-T_{\max }\right)\right) \\
+A_{l} \delta\left(\hat{T}-T_{\min }\right)+A_{r} \delta\left(\hat{T}-T_{\max }\right)
\end{array}
$$

that was first introduced by Lockwood and Naguib [8]. The symbol $\theta$ denotes the Heaviside function. The variables $A_{l}$ and $A_{r}$ correspond to the clipped parts and determine the magnitude of the delta pulses in order to satisfy the normalization condition. The clipping is necessary in order to avoid unphysical temperatures for high $\sigma_{T}$ values, i. e., negative temperatures or temperatures far above the adiabatic flame temperature.

For the species fluctuations, Girimaji [9] proposed the multivariate $\beta$-PDF:

$$
P_{Y}\left(\hat{Y}_{1}, \ldots, \hat{Y}_{N_{k}}\right)=\frac{\Gamma\left(\sum_{m=1}^{N_{k}} \beta_{m}\right)}{\prod_{m=1}^{N_{k}} \Gamma\left(\beta_{m}\right)}\left[\delta\left(1-\sum_{m=1}^{N_{k}} \hat{Y}_{m}\right) \prod_{m=1}^{N_{k}} \hat{Y}_{m}^{\beta_{m}-1}\right]
$$

with

$$
\beta_{m}=\tilde{Y}_{m} B
$$

Here,

$$
B=\frac{\sum_{m=1}^{N_{k}} \tilde{Y}_{m}\left(1-\tilde{Y}_{m}\right)}{\sigma_{Y}}-1
$$

where

$$
\sigma_{Y}=\sum_{m=1}^{N_{k}} \widetilde{Y_{m}^{\prime \prime 2}}
$$

is the sum of the species mass fraction variances. The delta function in Eq. (4) is required to fulfill the normalization condition of the PDF. Though the neglection of the full matrix of variances and covariances in this approach means a loss of information, the consideration of only the trace of the matrix which is $\sigma_{Y}$, leads to a significant reduction of computational time. When dealing with detailed chemistry, the number of components $N_{k}$ becomes quite large so that the solution 
of $n=(m-1)^{2} / 2+m$ transport equations $\left(m=N_{k}-1\right)$ becomes impossible for practical simulations.

In TASCOM3D, chemical reactions are treated fully coupled with the fluid motion in the finite-rate chemistry approach where the reaction rate $k_{r}$ is obtained from the modified Arrhenius equation:

$$
k_{r}=A_{r} T^{n_{r}} \exp \left(-\frac{E_{r}}{R_{m} T}\right)
$$

with the temperature $T$ and the gas constant $R_{m}$. The parameters $A_{r}, n_{r}$, and $E_{r}$ are available from a reaction mechanism which has to be chosen. For the simulations presented in this paper, the hydrogen oxidation scheme of Ó Conaire et al. [10] with 19 reactions and 8 species is used, because it is well validated, even for the high-pressure regime.

Equation (1) is solved on structured multiblock meshes via an implicit LowerUpper Symmetric Gauss-Seidel (LU-SGS) [11-13] finite-volume algorithm. Due to the recent work [14], the spatial discretization is of up to fifth order, whereas the temporal resolution is of up to third order. The code has been parallelized through MPI (Message Passing Interface) and is optimized to run on array processor architectures as well as massively parallel systems. TASCOM3D has been validated by a variety of combustion simulations ranging from subsonic [5] to supersonic [6] flows.

\section{TEST CASE}

Within the framework of the program "Focused Validation Data for Full Flow Staged Combustion (FFSC) Injectors" funded by NASA, a $\mathrm{GO}_{2} / \mathrm{GH}_{2}$ single element combustor (below called PennState preburner combustor) was examined at the Cryogenic Combustion Laboratory at the Pennsylvania State University. The specific interest in FFSC cycle engines arises from their simplicity and increased thrust-to-weight ratio, relative to other closed cycle engines.

The experiments reported by Marshall et al. [15] at several chamber pressures were designed to characterize the chamber wall heat transfer. Therefore, the circular combustion chamber was equipped with a large amount of Gordon type heat flux gauges and coaxial thermocouples. The axial positions of the temperature and heat flux measurements are illustrated in Fig. 1. The experiments investigated in this paper are those at the highest target chamber pressure of $5.17 \mathrm{MPa}$.

The chamber diameter is $38.1 \mathrm{~mm}$ and its length is $285.75 \mathrm{~mm}$. Two upstream preburners produce oxidizer-rich and fuel-rich gases, respectively. The oxidizerrich gas is fed to the combustion chamber through the inner tube of the coaxial injector with a diameter of $5.26 \mathrm{~mm}$ and is recessed $0.43 \mathrm{~mm}$ with respect to the 


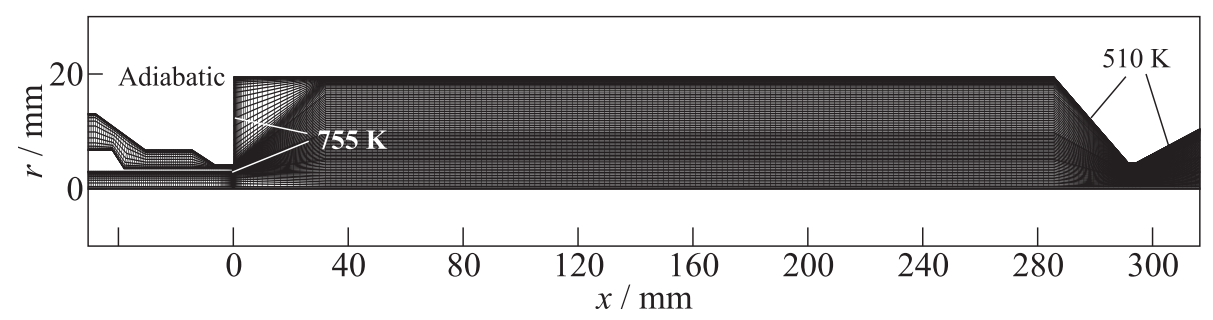

(a)

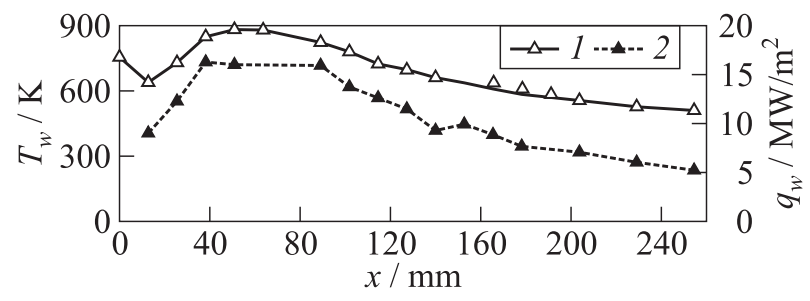

(b)

Figure 1 Computational domain $(a)$ and measured wall temperatures (1) and wall heat fluxes (2) [16] (b) for the PennState preburner combustor

combustion chamber face plane. The annular fuel feed has an inner diameter of $6.3 \mathrm{~mm}$ and an outer diameter of $7.49 \mathrm{~mm}$.

The operating conditions of the PennState preburner combustor are summarized in Table 1. It has to be noted that the $\mathrm{GO}_{2}$ and $\mathrm{GH}_{2}$ mass flow rates to the preburners were measured. Together with the measured preburner and combustion chamber pressures, the properties downstream of the preburners were calculated using the NASA chemical equilibrium code CEA and are provided

Table 1 Operating conditions of the PennState preburner combustor test case at a target pressure of $5.17 \mathrm{MPa}$ [16]

\begin{tabular}{|c|c|c|c|c|c|c|}
\hline Location & $\begin{array}{c}\text { Pressure, } \\
\mathrm{MPa}\end{array}$ & $\begin{array}{c}\text { Temperature } \\
\text { of products, } \\
\text { K }\end{array}$ & $\begin{array}{c}\mathrm{O}_{2} \\
\text { mass flow } \\
\text { in products, } \\
\mathrm{kg} / \mathrm{s}\end{array}$ & $\begin{array}{c}\mathrm{H}_{2} \\
\text { mass flow } \\
\text { in products, } \\
\mathrm{kg} / \mathrm{s}\end{array}$ & $\begin{array}{c}\mathrm{H}_{2} \mathrm{O} \\
\text { mass flow } \\
\text { in products, } \\
\mathrm{kg} / \mathrm{s}\end{array}$ & $\begin{array}{c}\text { Actual } \\
\text { pressure, } \\
\mathrm{MPa}\end{array}$ \\
\hline $\begin{array}{l}\text { Oxidizer } \\
\text { preburner }\end{array}$ & 5.85 & 700 & $8.55 \cdot 10^{-2}$ & 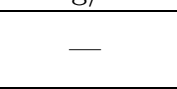 & $4.98 \cdot 10^{-3}$ & - \\
\hline Fuel preburner & 6.50 & 811 & - & $1.33 \cdot 10^{-2}$ & $1.98 \cdot 10^{-2}$ & - \\
\hline Main chamber & & & & & & 5.42 \\
\hline
\end{tabular}


in [16]. This test case has recently been of great interest and was simulated by numerous research groups using a variety of modeling approaches ranging from RANS to LES of different fidelity $[1,2]$.

In this work, the simulations are performed as unsteady $2 \mathrm{D}$ axisymmetric calculations on the computational domain illustrated in Fig. 1 with approximately 215000 volumes. Wherever possible, the measured temperatures are set as boundary conditions. The injector posttip wall is assumed to be isothermal with a temperature of $755 \mathrm{~K}$. For the combustion chamber wall, a temperature distribution corresponding to a least squares fit of the measured data points is set as boundary condition. The nozzle is water-cooled and has a temperature of $511 \mathrm{~K}$. All remaining walls are assumed to be adiabatic. The inflow conditions correspond to the values in Table 1. Unfortunately, no experimental data are available for turbulent quantities so that estimations had to be used.

All calculations were performed on the CRAY XE6 supercomputer at the High Performance Computing Center Stuttgart (HLRS). It is composed of 64bit AMD Opteron 6100 Series octocore processors at $2 \mathrm{GHz}$. Each computation was distributed over $256 \mathrm{MPI}$ jobs and performed with a constant timestep of $10 \mathrm{~ns}$. The spatial discretization was 5 th order while the time integration was 3rd order accurate. From the bulk velocity, the chamber flowthrough time can be estimated to be $8.3 \mathrm{~ms}$ [1]. To make sure that disturbances from the initial solution are washed out of the computational domain, preceding calculations were performed over approximately two flowthrough cycles. Subsequently, data averaging was carried out over $25 \mathrm{~ms}$, corresponding to approximately three flowthrough cycles. The computational consumption was approximately 25000 CPU hours and 32000 CPU hours for the calculation with laminar chemistry and assumed PDF approach, respectively.

\section{RESULTS}

Figure $2 a$ shows contour plots of the averaged $\mathrm{OH}$ mass fraction for the calculation without (top) and with (middle) the assumed PDF approach. The graph at the bottom shows an instantaneous hydroxyl distribution of the assumed PDF calculation. Note that all instantaneous contour plots in this section were extracted at the same simulation time. From the instantaneous contour plot, it can be clearly observed that the flame is anchored at the oxidizer posttip, although it appears to be lifted in the averaged graphs. A close-up look at the near-injector region in the averaged $\mathrm{OH}$ distribution, however, shows a region of high $\mathrm{OH}$ mass fraction right at the oxidizer posttip, indicating the flame base. The adjacent region with low hydroxyl mass fraction is due to strong corrugation of the flame just downstream the base that can be observed in the instantaneous contour 


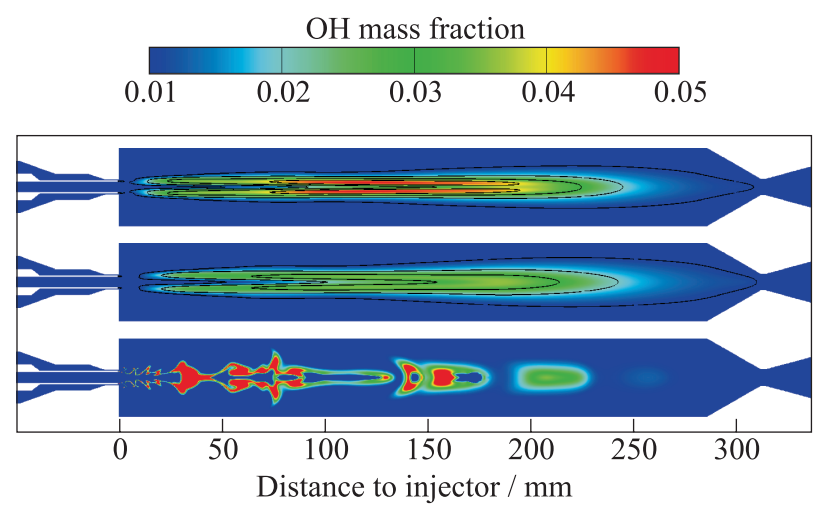

(a)

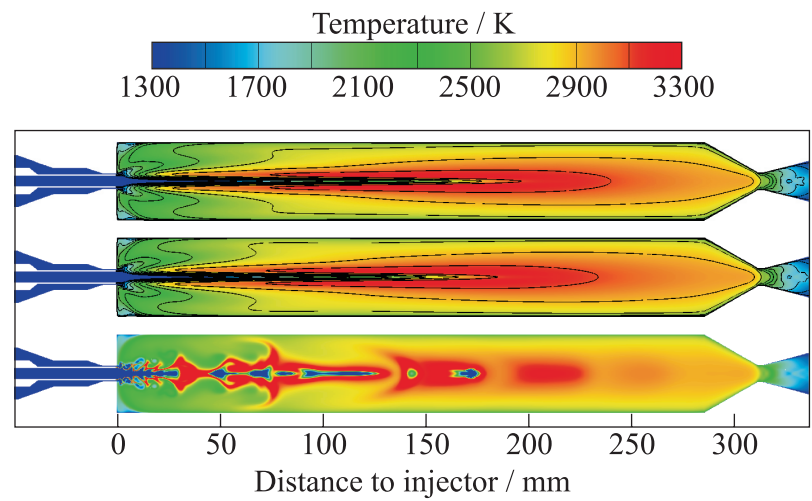

(b)

Figure 2 Hydroxyl and temperature contours of simulations with and without assumed PDF approach: $(a)$ averaged $\mathrm{OH}$ mass fraction contour with laminar chemistry (top) and assumed PDF approach (middle) and instantaneous $\mathrm{OH}$ distribution with assumed PDF approach at an arbitrary time step (bottom); and (b) averaged temperature contour with laminar chemistry (top) and assumed PDF approach (middle) and instantaneous temperature distribution with assumed PDF approach at an arbitrary time step (bottom).

plot. In the calculation with laminar chemistry, a region with high $\mathrm{OH}$ mass fraction stretches from approximately 80 to $200 \mathrm{~mm}$ downstream the injector. Here, averaged $\mathrm{OH}$ mass fraction values reach around $5 \%$.

When turbulence-chemistry interaction is considered in the simulation, the highest hydroxyl mass fractions in the same region are more than $20 \%$ lower and hardly reach a value of $4 \%$. Furthermore, the $\mathrm{OH}$ distribution is significantly broader towards the rear end of the combustion chamber. 
At the flame base that is anchored at the oxidizer posttip, however, both simulations compute averaged $\mathrm{OH}$ mass fraction of nearly $10 \%$. It has to be noted that the maximum instantaneous values of the hydroxyl mass fraction are also in this order of magnitude throughout the combustion chamber although the colour legend in Fig. $2 a$ is limited to a lower value.

Although the differences in the $\mathrm{OH}$ contour plots between the calculation with laminar and turbulent chemical source term are apparent, the differences in the temperature distributions are more subtle. The graphs in Fig. $2 b$ are arranged in the same way as those in Fig. $2 a$. Besides the observation that hot product gases are carried further upstream by the main recirculation zone in the assumed PDF calculation, there is hardly any difference between the two averaged contour plots. In both calculations, the highest average temperatures are in the region of $3500 \mathrm{~K}$. Moreover, due to the only moderate differences in the heat release in the combustion chamber, the flow field is more or less unaffected by utilizing the assumed PDF approach in the presented test case. In both simulations, two antipodal recirculation zones form in the upstream corner region of the combustion chamber. The main recirculation zone stretches from the injector face plane towards the combustion chamber wall at $150 \mathrm{~mm}$. It encloses the smaller counterrotating recirculation zone that forms in the corner and coincides with the region where the average temperature drops below $2100 \mathrm{~K}$. Due to the small differences between the calculations with laminar and turbulent chemical source term, it can be concluded that the combustion in this configuration of the PennState preburner combustor is quite close to equilibrium conditions. The instantaneous contour plot at the bottom again shows how strongly corrugated the flame is in the near injector region and how those structures merge and form larger patterns further downstream.

The fundamental principle of the assumed PDF approach is the solution of transport equations for the temperature variance and the sum of the species variances as introduced in Eqs. (3) and (4). From the perspective of physics, however, rather than the variance, the standard deviation is more tangible. Thus, actually, the square roots $\sqrt{\sigma_{T}}$ and $\sqrt{\sigma_{Y}}$ are plotted in Figs. $3 a$ and $3 b$, respectively.

Figure $3 a$ shows the standard deviation of temperature computed by the assumed PDF approach. The top graph represents the averaged distribution whereas an instantaneous distribution can be seen in the bottom graph. The highest average values of up to $800 \mathrm{~K}$ occur in the near injector region just downstream the oxidizer posttip. Overall, the region of elevated values between 300 and $600 \mathrm{~K}$ is reached approximately $180 \mathrm{~mm}$ into the combustion chamber. But also, in the vicinity of the combustion chamber walls, the values of more than $200 \mathrm{~K}$ can be reached. However, it has to be mentioned that the standard deviation decays in the boundary layer and vanishes directly at the wall. Also, in the upstream corners, a streak with standard deviations of around $200 \mathrm{~K}$ that separates the two recirculation zones can be observed. Comparing the 


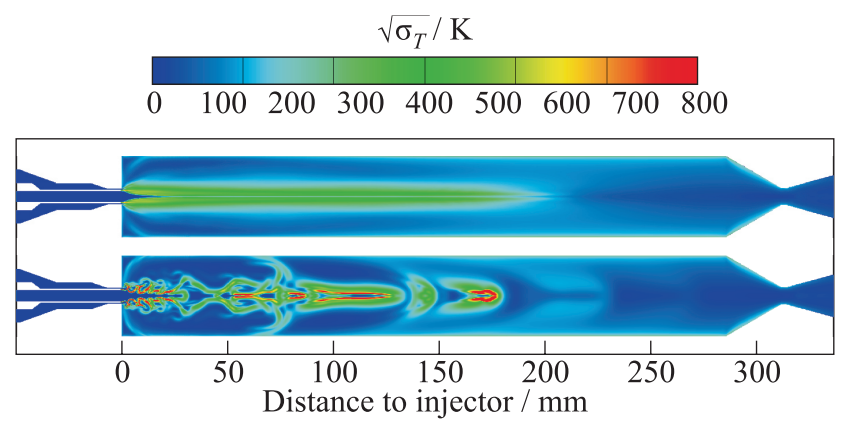

(a)

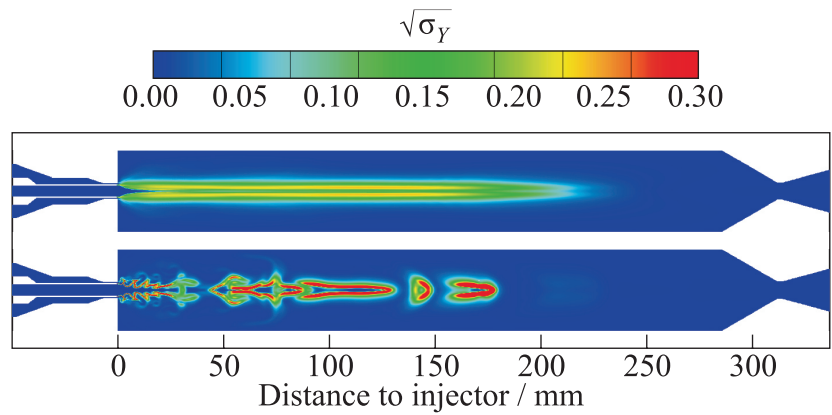

(b)

Figure 3 Contour plots of the modeling variables of the assumed PDF approach: averaged (top) and instantaneous (bottom) contour plot of $\sqrt{\sigma_{T}}(a)$; and $\sqrt{\sigma_{Y}}(b)$.

instantaneous graph with this of Fig. $2 b$, it can be observed that the highest standard deviations coincide with the regions of high-temperature gradients. In this regions, the instantaneous values of $\sqrt{\sigma_{T}}$ can reach $1000 \mathrm{~K}$ and more and they can occur even up until the middle of the combustor.

The overall shape of the $\sqrt{\sigma_{Y}}$ contour that is presented in Fig. $3 b$ shows a lot of similarity to the distribution of $\sqrt{\sigma_{T}}$. However, here, high values appear not only at the flame base but also in a region 100 to $160 \mathrm{~mm}$ downstream the injector close to the combustor symmetry axis. Furthermore, the region of elevated standard deviation values is reached further downstream into the combustor. On the other hand, no rise in $\sqrt{\sigma_{Y}}$ can be detected near the combustion chamber wall or in the recirculation zones. Maximum averaged and instantaneous values of $\sqrt{\sigma_{Y}}$ are approximately 0.3 and 0.6 , respectively.

As was mentioned earlier, the experimental data are available for the heat flux to the combustion chamber wall at discrete axial positions. In Fig. 4, these 


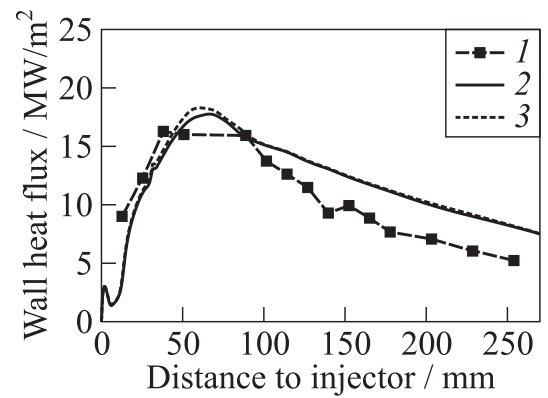

Figure 4 Comparison between the measured wall heat flux (1) and the simulations without (2) and with (3) assumed PDF modeling

data are compared to the wall heat flux data extracted from the simulations. In a previous paper [3], the simulations with laminar chemistry have been presented for the same test case. Due to a faulty temperature boundary condition in the older simulation, the wall heat fluxes differ from the ones shown in this paper. It can be stated that both simulations show quite good agreement with the experiment as far as the general shape of the distribution and the position of the maximum are concerned. Especially, the axial position of the initial steep rise is captured very accurately. The maximum heat flux is only slightly overpredicted by approximately $15 \%-20 \%$. At $100 \mathrm{~mm}$ downstream of the injector, however, the decline of the wall heat flux is not pronounced enough in the simulations so that the heat flux in this region is overpredicted with an almost constant offset of approximately $3 \mathrm{MW} / \mathrm{m}^{2}$. Due to the fact that the utilization of the assumed PDF approach has no major impact on the heat release and the flow field in the combustor, there is also no major difference in the wall heat flux between both simulations. Only between 30 and $70 \mathrm{~mm}$ downstream the injector, a slight deviation of up to $1 \mathrm{MW} / \mathrm{m}^{2}$ can be observed between the two calculations. The higher wall heat flux predicted by the simulation with assumed PDF approach is the result of the afore-mentioned stronger upstream transport of hot product gases.

Unfortunately, no experimental data are available for the standard deviation of the wall heat flux. Therefore, the simulation data for this quantity will also not be presented here. Yet, it should be mentioned that these results show only minor differences between the two simulations as well. In both cases, the maximum standard deviation of the wall heat flux is approximately $3.5 \mathrm{MW} / \mathrm{m}^{2}$ and coincides with the position of the maximum average wall heat flux at around $60 \mathrm{~mm}$ downstream the injector.

In the experiment, a combustion chamber pressure of $5.42 \mathrm{MPa}$ was measured $12.7 \mathrm{~mm}$ downstream the injector face plane which is slightly higher than 
the target pressure of $5.17 \mathrm{MPa}(750 \mathrm{psi})$. In the simulations, however, the chamber pressure at this position lies around $9 \%$ lower at 4.96 and $4.94 \mathrm{MPa}$ for the simulation with laminar chemistry and with assumed PDF approach, respectively.

\section{CONCLUDING REMARKS AND OUTLOOK}

A multivariate assumed PDF approach has been successfully used for the unsteady RANS simulation with finite-rate chemistry of the PennState preburner combustor. To the authors' knowledge, this is the first such attempt in the simulation of rocket combustors. The rise in computational cost compared to the calculations with laminar chemistry was found to be moderate (approximately $40 \%-50 \%$ ). The presented simulations are able to produce a good representation of the wall heat flux measured in the experiment. Merely in the downstream region, larger deviations can be observed. Yet, for the design of the cooling system of the combustors, the knowledge about the position and the magnitude of the maximum wall heat flux are most important. This information is reproduced quite accurately by both simulations, with a slight overprediction of the maximum by less than $20 \%$. Bearing in mind the difficulties of performing wall heat flux measurements, this constitutes a feasible value for engineering purposes.

The PennState preburner test case was also subject of the work presented in [1] where a variety of approaches of different fidelity, ranging from RANS to LES, was employed. This broad investigation showed that even the use of more time consuming LES models does not guarantee an excellent agreement with the experimental heat flux data. Only the model of highest fidelity (3D LES with finite-rate chemistry - computational time: 2 million CPU hours) reproduced the wall heat flux accurately. Also, in this light, the results in this work, obtained with URANS and therefore, much less computational resources, appear quite satisfactory. However, the mentioned state-of-the-art LES also showed certain $3 \mathrm{D}$ effects that can naturally not be captured in a $2 \mathrm{D}$ axisymmetric calculation. Therefore, a 3D URANS simulation is necessary for further investigation and currently under preparation.

The utilization of the PDF approach leads to observable changes in the distribution of the radical distributions. This has been shown in Fig. $2 a$ for the hydroxyl molecule but is also true for atomic hydrogen and oxygen. The heat release and flow field, however, are more or less unaffected by these changes. Therefore, the differences in the computed wall heat fluxes are also marginal. Only in the region of maximum wall heat fluxes, a small deviation between the two simulations can be observed. The higher heat flux in the simulation considering turbulence-chemistry interaction was found to result from a stronger 
upstream transport of hot product gases in the recirculation zone. The fact that the assumed PDF approach only yields small changes indicates that the presented combustor configuration seemingly operates close to equilibrium conditions. This result is unexpected and requires further investigation. A meaningful analysis of this aspect, however, is only possible with detailed knowledge of the actual PDFs of the temperature and species mass fractions throughout the combustion chamber. Such information can only be obtained from extensive experiments with optical access to the combustion chamber or a numerical simulation incorporating a transported PDF approach. Currently, there is work under way to pursuit such a numerical investigation.

\section{ACKNOWLEDGMENTS}

This ongoing investigation is funded by the German Research Foundation (DFG) within the framework of the research program SFB-TRR 40. The support is greatly appreciated.

The simulations are performed at the HLRS. The authors wish to thank HLRS for the computing resources and the technical support.

\section{REFERENCES}

1. Tucker, K., S. Menon, C. Merkle, J. C. Oefelein, and V. Yang. 2008. Validation of high-fidelity CFD simulations for rocket injector design. AIAA Paper No. 20085226 .

2. Lian, C., and C. L. Merkle. 2010. Contrast between steady and time-averaged unsteady combustion simulations. AIAA Paper No. 2010-371.

3. Lempke, M., P. Gerlinger, M. Rachner, and M. Aigner. 2011. Steady and unsteady RANS simulations of cryogenic rocket combustors. AIAA Paper No. 2011-101.

4. Pope, S. B. 1985. PDF methods for turbulent reactive flows. Prog. Energy Combust. Sci. 11:119-92.

5. Gerlinger, P. 2003. Investigations of an assumed pdf approach for finite-ratechemistry. Combust. Sci. Technol. 175(5):841-72.

6. Gerlinger, P., P. Stoll, M. Kindler, F. Schneider, and M. Aigner. 2008. Numerical investigations of mixing and combustion enhancement in supersonic combustors by strut induced streamwise vorticity. Aerospace Sci. Technol. 12(2):159-68.

7. Wilcox, D. C. 2008. Formulation of the $\mathrm{k}-\omega$ turbulence model revisited. AIAA J. 46:2823-38.

8. Lockwood, F. C., and A.S. Naguib. 1975. The prediction of the fluctuations in the properties of free, round-jet, turbulent, diffusion flames. Combust. Flame 24:10924 . 
9. Girimaji, S. S. 1991. A simple recipe for modeling reacting-rates in flows with turbulent combustion. AIAA Paper No. 1991-1792.

10. Ó Conaire, M., H. J. Curran, J. M. Simmie, W. J. Pitz, and C. K. Westbrook. 2004. A comprehensive modeling study of hydrogen oxidation. Int. J. Chem. Kinetics 36(11)603-22.

11. Jameson, A., and S. Yoon. 1987. Lower-upper implicit scheme with multiple grids for the Euler equations. AIAA J. 25(7):929-37.

12. Shuen, J.S. 1992. Upwind differencing and LU factorization for chemical nonequilibrium Navier-Stokes equations. J. Comput. Phys. 99(2):233-50.

13. Gerlinger, P., H. Möbus, and D. Brüggemann. 2001. An implicit multigrid method for turbulent combustion. J. Comput. Phys. 167(2):247-76.

14. Gerlinger, P. 2012. High-order multi-dimensional limiting for turbulent flows and combustion. J. Comput. Phys. 231(5):2199-228.

15. Marshall, W., S. Pal, R. Woodward, and R. Santoro. 2005. Benchmark wall heat flux data for a $\mathrm{GO}_{2} / \mathrm{GH}_{2}$ single element combustor. AIAA Paper No. 2005-3572.

16. Pal, S., W. Marshall, R. Woodward, and R. Santoro. 2006. Wall heat flux measurements in a uni-element $\mathrm{GO}_{2} / \mathrm{GH}_{2}$ shear coaxial injector. 3rd Workshop (International) on Rocket Combustion Modeling Proceedings. Vernon, France. 